IAU Colloquium 164: Radio Emission from Galactic and Extragalactic Compact Sources

ASP Conference Series, Vol. 144, 1998

J. A. Zensus, G. B. Taylor, \& J. M. Wrobel (eds.)

\title{
Limiting Brightness Temperature for Synchrotron Sources
}

\author{
A. Lähteenmäki \\ Metsähovi Radio Research Station, Helsinki University of Technology, Finland \\ E. Valtaoja \\ Tuorla Observatory, University of Turku, Finland
}

\begin{abstract}
The maximum brightness temperature limit for synchrotron sources derived from inverse Compton catastrophe is usually taken to be $10^{12} \mathrm{~K}$. Readhead has argued in 1994 that the equipartition brightness temperature may be a better estimate for $T_{b, l i m}$. It provides a value of $5 \times 10^{10} \mathrm{~K}$. We suggest that a reasonable estimate of the value of $T_{b, \text { lim }}$ can also be achieved by comparing Doppler boosting factors from total flux density variations at 22 and $37 \mathrm{GHz}$ with traditional Doppler factors from the SSC X-ray flux. We also compare our variability brightness temperatures with values calculated for individual sources observed with VLBI, obtaining other independent estimates of $T_{b, l i m}$.
\end{abstract}

The almost twenty years of continuum monitoring of active galaxies in Metsähovi has resulted in an extensive database at 22 and $37 \mathrm{GHz}$. By using this unique high frequency total flux density variation data we have calculated the intrinsic variability brightness temperature and the Doppler boosting factor for each source (almost 80 sources out of the total of $\sim 130$ sources). We compared the total flux density variation brightness temperatures at $22 \mathrm{GHz}$ with brightness temperatures from simultaneous VLBI observations. We had three different VLBI data sets: the VSOP Pre-Launch Survey (PLS) (Moellenbrock et al. 1996), Bloom et al. (1997), and Wiik \& Valtaoja, (these Proceedings, p. 151). We found that the variability and VLBI values correlate with each other with probability $P_{\text {Spearman }}<0.00005$. Due to different dependencies on the Doppler factor, $T_{b, l i m}$ can be estimated.

Next we compared our variability Doppler factors with the SSC Doppler factors from Güijosa \& Daly (1996) (Fig. 1). The data are not simultaneous but we rather used the highest observed brightness temperature for each source. The variability and SSC values are highly correlated with probability $P_{\text {Spearman }}=0.0002$. Assuming that the variability and SSC Doppler factors are equal, the limiting brightness temperature $T_{b, l i m}$ can be calculated (Valtaoja, these Proceedings, p. 35). We did the comparison also for 3C 345 for which the estimated range of Doppler boosting factors is $5.5 \leq D \leq 10.5$ (Unwin et al. 1994). Figure 2 shows the distribution of $T_{b, l i m}$ for each data set. The median values are:

- $10.82 \pm 0.41$ for $3 \mathrm{C} 345$

- $10.41 \pm 0.43$ for Wiik $(N=5)$

- $10.89 \pm 0.17$ for Bloom et al. $(N=10)$

- $11.24 \pm 0.10$ for PLS $(N=33)$

- $11.30 \pm 0.20$ for Güijosa \& Daly $(N=48)$.

The limiting brightness temperatures derived from all the different data sets above imply a value of $T_{b, l i m} \simeq 10^{11} \mathrm{~K}$ noticeably lower than the usual $10^{12} \mathrm{~K}$ and in good accordance with Readhead. 


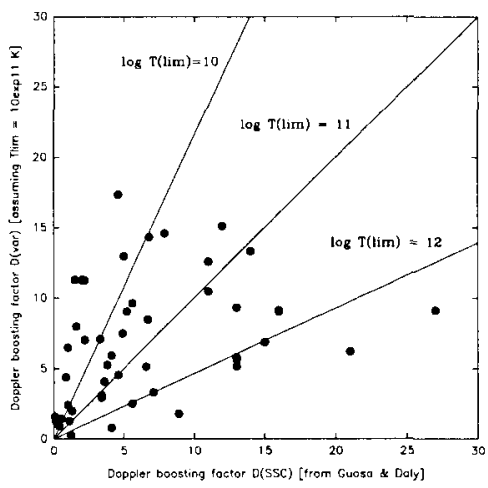

Figure 1. The Doppler boosting factors derived from variability brightness temperatures vs. the SSC-derived boosting factors.

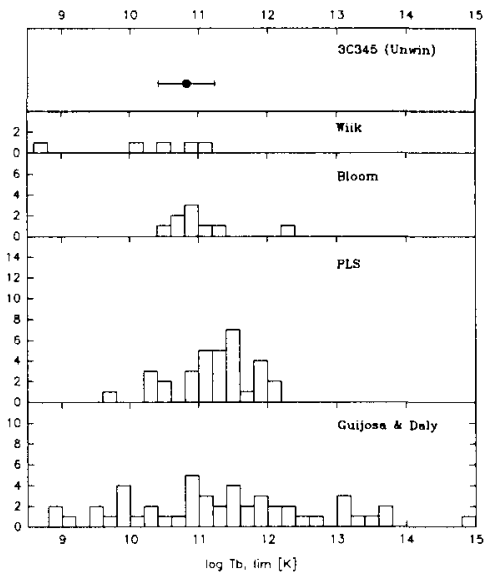

Figure 2. Limiting brightness temperatures calculated from various data sets (see text for details).

\section{References}

Bloom, S. D., et al. 1997. ApJS, preprint.

Güijosa, A., \& Daly, R. A. 1996. ApJ, 461, 600-608.

Moellenbrock, G. A., et al. 1996. $A J, 111,2174-2186$.

Readhead, A. C. S. 1994. ApJ, 426, 51-59.

Unwin, S. C., et al. 1994. ApJ, 432, 103-113. 